\title{
ON THE CONTINUITY OF HAAR MEASURE ON TOPOLOGICAL GROUPOIDS
}

\author{
ANTHONY KAREL SEDA
}

\begin{abstract}
It is shown that continuity of a family of invariant (Haar) measures on a topological groupoid $G$ is equivalent to the continuity of the implied convolution product $f * g$ for all pairs of functions $f$ and $g$. An example is given of a groupoid which admits no (continuous) Haar measure. It results, therefore, that the usual $C^{*}$-algebra associated with a Haar measure on $G$ cannot, in general, be constructed. Some remarks are included concerning the construction of Haar measures on the holonomy groupoid of a foliated manifold.
\end{abstract}

1. Introduction. Several definitions of invariant, or Haar measure have been investigated in the literature for locally compact topological groupoids $G$, and chronologically they appeared in the following order. First, Westman [17] proposed a definition for locally trivial $G$ in which positive Radon measures $\mu_{x y}$ are assigned to the morphism sets $G(x, y)$, one for each pair $(x, y)$ of objects of $G$. These measures are subject to suitable invariance and smoothness conditions in the nature of (I) and (II) below, called respectively "left invariance" and "continuity". In [16] he gave a somewhat different definition for a principal groupoid; in this case positive Radon measures $\mu_{x}$ are given, indexed by the object set $X$ of $G$, defined on $G$ and supported by the sets $G^{x}=\bigcup_{y \in X} G(y, x)$. Again, invariance and continuity conditions are imposed. Of course, invariance here means invariance with respect to (left) multiplication in $G$. Thus, if $\alpha \in G$ and the range $\operatorname{r}(\alpha)$ of $\alpha$ is $y$ and the domain $\mathrm{d}(\alpha)$ of $\alpha$ is $x$, then $\alpha \beta$ is defined if and only if $\operatorname{r}(\beta)=x$, i.e., if and only if $\beta \in G^{x}$. For this reason, the sets $G^{x}$ are the natural carriers of any measures to be designated "invariant". This point of view was adopted by us in [9 and 11] in our formulation of a Haar measure for arbitrary $G$.

The distillate of all these notions is the following definition: A Haar Measure on $G$ is a family $\left\{\mu_{x} ; x \in X\right\}$ of positive Radon measures defined on $G$ with $\operatorname{supp}\left(\mu_{x}\right)=G^{x}$ for each $x$, and satisfying

(I) $\int_{G} f^{\alpha} d \mu_{r(\alpha)}=\int_{G} f d \mu_{d(\alpha)}$ for all $\alpha \in G$ and $f \in \mathscr{K}(G)$, where $f^{\alpha}(\beta)=f\left(\alpha^{-1} \beta\right)$.

(II) For all $f \in \mathscr{K}(G)$, the function $x \mapsto \int_{G} f d \mu_{x}$, mapping $X$ into $C$, is continuous.

Here $\mathscr{K}(G)$ denotes the space of all continuous complex valued functions on $G$ with compact support.

This is the definition adopted by Renault in [7] and is the one now in general use, see $[2,3,4,5,6,8$ and 15$]$.

Received by the editors December 6, 1984 and, in revised form, February 4, 1985.

1980 Mathematics Subject Classification. Primary 46L99: Secondary 28C10. 
Of course, (II) simply states that the mapping $M(x)=\mu_{x}$ is continuous on $X$ when the cone $\mathscr{M}_{+}(G)$ of positive Radon measures on $G$ is endowed with the vague topology. It is not yet known exactly what restrictions are placed on $G$ by condition (II), although (II) actually follows from (I) under the workable hypothesis that each of the maps $d: G^{x} \rightarrow X$ is open, see [10].

Aside from developments made in Mackey's theory of virtual groups and ergodic groupoids, see the bibliography of [7], Haar measures can be used to perform certain averaging operations, familiar in the group context, and to construct the regular representation of $G$, see [12 and 13]. It is, however, the associated convolution algebras and resulting $C^{*}$-algebras of $G$ which have attracted most attention. These have been extensively developed by Renault in [7]. In particular, by means of the holonomy groupoid, one obtains the $C^{*}$-algebra of a foliated manifold $(M, \mathscr{F})$ initiated by Connes [1], see also $[2,4,6,8,15]$, and the $C^{*}$-algebra of a foliated bundle [5]. In such applications, condition (II) is crucial in that it ensures the desired continuity of the end-result, depending on the context. For example, if $\sigma$ is a continuous 2-cocycle on $G^{2}$ (the set of composable pairs in $G$ ) with values in the circle group $T$, then the convolution product $f * g$ of functions $f$ and $g$ in $\mathscr{K}(G)$ is defined as follows, see [7, Chapter II]:

$$
f * g(\alpha)=\int_{G} f(\alpha \beta) g\left(\beta^{-1}\right) \sigma\left(\alpha \beta, \beta^{-1}\right) d \mu_{d(\alpha)}(\beta) .
$$

In this case, (II) is sufficient to ensure that $f * g$ is continuous for all $f$ and $g$.

The purpose of this note is fourfold. First, we show that the definition given above of a Haar measure is "best possible" in that condition (II) cannot be replaced by anything weaker:

TheOREM. Let $\left\{\mu_{x} ; x \in X\right\}$ be a family of positive Radon measures on $G$, with $\operatorname{supp}\left(\mu_{x}\right)=G^{x}$ for each $x$, satisfying (I). Then the following two conditions are equivalent.

(a) The mapping $M: X \rightarrow \mathscr{M}_{+}(G)$ defined by $M(x)=\mu_{x}$ is vaguely continuous.

(b) Given any continuous 2-cocycle $\sigma, f * g$ is continuous for all $f$ and $g$ in $\mathscr{K}(G)$.

Second, we record below some remarks which greatly simplify the construction of a Haar measure in certain special cases including the holonomy groupoid.

Third, we give in $\$ 3$ a simple example of a topological groupoid $G$ which does not possess any Haar measure in our present sense. It is of course (II) which fails here, and this counterexample settles what has been an open problem for several years. This is unfortunate in that it results from the main theorem that it is not therefore possible to construct the $C^{*}$-algebra (in accordance with [7]) of a groupoid in general.

Finally, we collect together a fairly extensive bibliography covering some of the recent developments made in this area.

Actually this definition of a Haar measure is the minimum that one can postulate in practice, for it is usually necessary to have a Radon measure $\mu$ to hand on $X$, see [7, Chapter II]. As a matter of fact, each measure $\mu_{x}$ determines uniquely a measure 
(class) on $X$ in terms of which it is an integral of "translates" of Haar measure on the vertex group $G\{x\}=G(x, x)$, at least with suitable separability conditions on $G$, see [11, Theorem 2]. Such a $\mu$ needs to be suitably related to the $\mu_{x}$, see [11, §2], but in the presence of (II) can always be chosen arbitrarily. This is so in the important situation when $G$ is locally trivial, for in this particular case the condition of [10] on the maps $d: G^{x} \rightarrow X$ is satisfied and so (II) is automatic from (I). Furthermore, each of the sets $G^{x}$ is a locally trivial principal bundle with structure group $G\{x\}$, see $[11, \S \S 3,4]$. The charts of $G^{x}$ can thus be used to construct $\mu_{x}$ in terms of a measure $\mu$ on $X$ and a Haar measure $\nu$ on $G\{x\}$. Indeed, if $G\{x\}$ is unimodular, $\mu_{x}$ is then actually a "product" of $\mu$ and $\nu$ in the bundle $G^{x}$. It follows therefore that the support of $\mu_{x}$ equals $G^{x}$ if and only if the support of $\mu$ equals $X$.

All these facts are worth noting because they apply to the holonomy groupoid of $(M, \mathscr{F})$ which is of course locally trivial.

My thanks are due to the referee for making some improvements to this paper and in particular for suggesting a proof of an unpublished result in [16] used here in the proof of the corollary to the main theorem.

2. The proof of the theorem. We begin with two lemmas, the first of which is due to Westman [18, Theorem 5.1], and is often useful in practice.

LEMMA 1. Let $G$ be a topological groupoid and let $X$ be endowed with the subspace topology of $G$, as usual. Then the mappings $r$ and $d$ of $G$ onto $X$ are quotient mappings.

Lemma 2. Suppose $\delta: G \times G \rightarrow C$ is a continuous function and $M: G \rightarrow \mathscr{M}_{+}(G)$, $M(\alpha)=\mu_{\alpha}$, is vaguely continuous. Let $\delta_{\alpha}: G \rightarrow C$ denote the function defined for each $\alpha \in G$ by $\delta_{\alpha}(\beta)=\delta(\alpha, \beta)$, and let $\lambda_{\alpha}=\delta_{\alpha} \mu_{\alpha}$ denote the product of the Radon measure $\mu_{\alpha}$ by the continuous function $\delta_{\alpha}$. Then the mapping $\Lambda: G \rightarrow \mathscr{M}_{+}(G)$ defined by $\Lambda(\alpha)=\lambda_{\alpha}$ is also vaguely continuous.

Proof. Let $\alpha_{0} \in G$ and $f \in \mathscr{K}(G)$ be arbitrary. Let $U$ be a compact neighbourhood of $\alpha_{0}$ in $G$ and $\Phi \in \mathscr{K}(G)$ be nonnegative and such that $\Phi \equiv 1$ on the compact set $K=\operatorname{supp}(f)$; then $\sup _{\alpha \in U}\left\|\Phi \mu_{\alpha}\right\|<+\infty$ by [14, Proposition 1]. Hence for $\alpha \in U$ we have

$$
\begin{aligned}
& \left|\lambda_{\alpha}(f)-\lambda_{\alpha_{0}}(f)\right|=\left|\mu_{\alpha}\left(\delta_{\alpha} f\right)-\mu_{\alpha_{0}}\left(\delta_{\alpha_{0}} f\right)\right| \\
& \quad=\left|\mu_{\alpha}\left(\delta_{\alpha} f-\delta_{\alpha_{0}} f\right)+\mu_{\alpha}\left(\delta_{\alpha_{0}} f\right)-\mu_{\alpha_{0}}\left(\delta_{\alpha_{0}} f\right)\right| \\
& \quad \leqslant\left|\int_{K}\left(\delta_{\alpha}-\delta_{\alpha_{0}}\right)(\beta) f(\beta) \Phi(\beta) d \mu_{\alpha}(\beta)\right|+\left|\mu_{\alpha}\left(\delta_{\alpha_{0}} f\right)-\mu_{\alpha_{0}}\left(\delta_{\alpha_{0}} f\right)\right| \\
& \quad \leqslant \sup _{\beta \in K}\left|\left(\delta_{\alpha}-\delta_{\alpha_{0}}\right)(\beta)\right|\|f\| \sup _{\alpha \in U}\left\|\Phi \mu_{\alpha}\right\|+\left|\mu_{\alpha}\left(\delta_{\alpha_{0}} f\right)-\mu_{\alpha_{0}}\left(\delta_{\alpha_{0}} f\right)\right| .
\end{aligned}
$$

Both terms on the right-hand side tend to zero with $\alpha \rightarrow \alpha_{0}$ in $U$, and the lemma is established.

REMARK. This lemma is valid for arbitrary $G$ for at no point in the proof is the groupoid structure invoked. 
The proof of the theorem now proceeds as follows.

(a) $\Rightarrow$ (b). This is established in [7, Chapter II] and [14].

(b) $\Rightarrow$ (a). By making the transformation $\beta \mapsto \alpha^{-1} \beta$ and using the invariance (I), $f * g$ can be cast in the form

$$
f * g(\alpha)=\int_{K} f(\beta) g\left(\beta^{-1} \alpha\right) \sigma\left(\beta, \beta^{-1} \alpha\right) d \mu_{\alpha}(\beta)
$$

where $K=\operatorname{supp}(f)$, and $\mu_{\alpha}=\mu_{r(\alpha)}$ for all $\alpha$. Thus

$$
f * g(\alpha)=\int_{K} f(\beta) g\left(\beta^{-1} \alpha\right) \delta_{\alpha}(\beta) d \mu_{\alpha}(\beta)
$$

where $\delta(\alpha, \beta)=\sigma\left(\beta, \beta^{-1} \alpha\right)$. So it suffices to suppose that $\sigma \equiv 1$, for if it is established that $\alpha \mapsto \delta_{\alpha} \mu_{\alpha}$ is vaguely continuous, then the effect of Lemma 2 (applied to the set $G^{2}$ of composable pairs in $G \times G$ ) is that $\alpha \mapsto \delta_{\alpha}^{-1}\left(\delta_{\alpha} \mu_{\alpha}\right)$ is vaguely continuous, i.e. that $\alpha \mapsto \mu_{\alpha}$ is vaguely continuous, and vice-versa.

Now let $\alpha \in G$ and let $U$ be a compact neighbourhood of $\alpha$ in $G$. Let $C$ be the compact set which is the image under the composition in $G$ of $\left(K^{-1} \times U\right) \cap G^{2}$, where $K^{-1}$ is the image of $K=\operatorname{supp}(f)$ under the inverse map in $G$; let $\Phi \in \mathscr{K}(G)$ be nonnegative and such that $\Phi \equiv 1$ on $C$. Then, if $g$ is any continuous function on $G, \Phi g \in \mathscr{K}(G)$ and $\Phi g=g$ on $C$. From this it easily follows that

$$
f * g=f *(\Phi g)
$$

on $U$. From this and the hypothesis, it in turn follows that $f * g$ is defined and continuous on $G$ for any $f \in \mathscr{K}(G)$ and any continuous $g$. Consequently, we are at liberty to set $g \equiv 1$ on $G$ to conclude that the function $\int_{G} f(\beta) d \mu_{\alpha}(\beta)$ is continuous in $\alpha$ or, in other words, that $\alpha \mapsto \mu_{\alpha}$ is vaguely continuous. This latter mapping is the composite $M \circ r$, and so the conclusion follows from Lemma 1 and the universal property of quotients.

COROLlaRY. If condition (b) of the theorem above is satisfied, then $r$ and $d$ are open mappings of $G$ onto $X$.

Proof. By the main theorem it suffices to show that (a) implies the conclusion. This latter fact, that is, that (a) implies that the maps $r$ and $d$ are open was stated without proof as Proposition I.4 of [16]. For the reader's convenience we record below, in our present notation, a proof, due to the referee, of Westman's result.

Let $\alpha \in G$ and let $U$ be a nonempty compact neighbourhood of $\alpha$ in $G$. By Urysohn's lemma there is a nonnegative $f \in \mathscr{K}(G)$ with $f(\alpha)$ positive and $\operatorname{supp}(f)$ contained in $U$. Letting $V$ denote the interior of $\operatorname{supp}(f)$, we must show that $W=r(V)$ is an open neighbourhood of $y=r(\alpha)$. But this is immediate from the fact that $\operatorname{supp}\left(\mu_{x}\right)=G^{x}$ for each $x$ and, hence, that $\mu_{x}(f)$ is positive if and only if $x \in W$.

3. A counterexample. Let $X$ denote the closed unit interval $[0,1]$ in the $x$-axis and let $T$ denote the unit circle in the $y z$-plane in $R^{3}$. Then $H=X \times T$ is a topological groupoid in the usual way. Moreover, the object space of $H$ can be identified with $X$, and $H^{x}=H\{x\}$ is a copy of $T$ for each $x$. Let $G$ be the subgroupoid of $H$ 
obtained by replacing $H^{x}$ by the trivial group $G^{x}=G\{x\}=\{(x, 1,0)\}$ for all $x>\frac{1}{2}$. Clearly, $G$ is a compact Hausdorff topological groupoid. We show here that, whilst $G$ admits many families $\left\{\mu_{x} ; x \in X\right\}$ of measures satisfying (I), it admits no Haar measure.

Suppose on the contrary that $\left\{\mu_{x} ; x \in X\right\}$ is a Haar measure on $G$. Then $\mu_{x}$ is a Haar measure on the group $G\{x\}$ for each $x$, and in fact is a weight attached to $(x, 1,0)$ for all $x>\frac{1}{2}$. Putting $f \equiv 1$ on $G$, we conclude that the function $x \mapsto \mu_{x}\left(G^{x}\right)$ is continuous. Dividing by this positive function, we see that $\left\{\nu_{x} ; x \in X\right\}$ is a Haar measure also, where, for each $x \in X, \nu_{x}=\mu_{x} / \mu_{x}\left(G^{x}\right)$.

Moreover, $\nu_{x}\left(G^{x}\right)=1$ for all $x$, and so we can and will assume from the outset that the $\mu_{x}$ are normalised. Now select a continuous function $f$ on $H$ with values in $[0,1]$ such that $f(x, 1,0)=1$ and

$$
\int_{H^{x}} f d \lambda_{x} \leqslant \frac{1}{2}
$$

for all $x \in X$, where $\lambda_{x}$ denotes Lebesgue measure on $H^{x}$. Since $\mu_{x}$ coincides with $\lambda_{x}$ if $x \leqslant \frac{1}{2}$, we now have

$$
\mu_{x}(f) \leqslant \frac{1}{2} \quad \text { if } x \leqslant \frac{1}{2} \quad \text { and } \quad \mu_{x}(f)=1 \quad \text { if } x>\frac{1}{2} .
$$

Thus, the function $x \mapsto \mu_{x}(f)$ is discontinuous at $x=\frac{1}{2}$; and this yields the desired contradiction.

REMARK. In this example, $r$ and $d$ are not open mappings of $G$ onto $X$. Moreover, $G$ is totally disconnected in the sense that $G(x, y)=\varnothing$ if $x \neq y$. This case is at the opposite extreme, therefore, to that of [10] where $G$ is necessarily locally transitive if each of the maps $d: G^{x} \rightarrow X$ is open. Furthermore, the condition (A) that each of the maps $d: G^{x} \rightarrow X$ be open implies the condition (B) that the mapping $d: G \rightarrow X$ be open. Necessary and sufficient conditions for the existence of a Haar measure on a groupoid $G$ lie, therefore, somewhere between the necessary one (B) and the sufficient one (A). They do not, however, coincide with (A) since the groupoid $H$ in the example above fails to satisfy (A) but does admit many Haar measures.

\section{REFERENCES}

1. Alain Connes, Sur la théorie non-commutative de l'intégration, Lecture Notes in Math., vol. 725 , Springer-Verlag, Berlin and New York, 1978, pp. 19-143.

2. T. Fack and G. Skandalis, Sur les représentations et idéaux de la $C^{*}$-algèbre d'un feuilletage, J. Operator Theory 8 (1982), 95-129.

3. P. S. Muhly and J. Renault, $C^{*}$-algebras of multivariable Wiener-Hopf operators, Trans. Amer. Math. Soc. 274 (1982), 1-44.

4. T. Natsume and H. Takai, Connes algebras associated to Reeb foliations, Preprint, Saitama and Tokyo Metropolitan Universities, Japan, 1981. $C^{*}$-News, No. 14.

5. Connes algebras associated to foliated bundles, Preprint, Saitama and Tokyo Metropolitan Universities, Japan, 1981. C*-News, No. 16.

6. Moto O'uchi, Coverings of foliations and associated $C^{*}$-algebras, Preprint, Ehime University, Japan. $C^{*}$-News, No. 20.

7. J. Renault, A groupoid approach to $C^{*}$-algebras, Lecture Notes in Math., vol. 793, Springer-Verlag, Berlin and New York, 1980. , $C^{*}$-algebras of groupoids and foliations, Operator Theory and Applications, Part 1 (R. V. Kadison, ed.), Proc. Sympos. Pure Math., vol. 38, Amer. Math. Soc., Providence, R.I., 1982, pp. 339-350. 
9. A. K. Seda, Un concept de mesures invariantes pour les groupoides topologiques, C. R. Acad. Sci. Paris Sér. A 280 (1975), 1603-1605.

10. , A continuity property of Haar systems of measures, Ann. Soc. Sci. Bruxelles 89 (1975), 429-433.

11. __ Haar measures for groupoids, Proc. Roy. Irish Acad. Sect. A 76 (1976), 25-36.

12. _ Quelques résultats dans la Catégorie des groupoids d'opérateurs, C. R. Acad. Sci. Paris Sér. A 288 (1979), 21-24.

13. , Banach bundles and a theorem of J. M. G. Fell, Proc. Amer. Math. Soc. 83 (1981), 812-816.

14. Banach bundles of continuous functions and an integral representation theorem, Trans. Amer. Math. Soc. 270 (1982), 327-332.

15. H. Takai, $C^{*}$-algebras of Anosov foliations, Preprint, Univ. of Warwick, U. K., 1983. $C^{*}$-News, No. 19.

16. J. J. Westman, Nontransitive groupoid algebras, Univ. of California at Irvine, 1967.

17. ___ Harmonic analysis on groupoids, Pacific J. Math. 27 (1968), 621-632.

18. Groupoid theory in algebra, topology and analysis, Univ. of California at Irvine, 1971.

Department of Mathematics, University College, Cork, Ireland 